\title{
Heterotopia pancreática en pólipo gástrico. Presentación de caso
}

\section{A case of heterotopic pancreas in a gastric polyp}

\author{
Pedro Rosales Torres, ${ }^{1 *}$ Rafael Pila Pérez, ${ }^{2}$ Pedro León Acosta, ${ }^{3}$ Rafael Pila Peláez. ${ }^{4}$
}

\footnotetext{
1 Especialista de primer grado en Anatomía Patológica. Instructor. Hospital Provincial Docente Manuel Ascunce Domenech. Camagüey, Cuba.

2 Especialista de segundo grado en Medicina Interna. Profesor titular. Hospital Provincial Docente Manuel Ascunce Domenech. Camagüey, Cuba.

3 Especialista de primer grado en Medicina Interna. Instructor. Hospital Provincial Docente Manuel Ascunce Domenech. Camagüey, Cuba.

${ }^{4}$ Especialista de segundo grado en Medicina Interna. Profesor auxiliar. Hospital Provincial Docente Manue Ascunce Domenech. Camagüey, Cuba.
}

*Correspondencia: rtpedro.cmw@infomed.sld.cu.

Fecha recibido: $22 / 02 / 18$

Fecha aceptado: 09/05/18

\begin{abstract}
Resumen
La heterotopia pancreática es un hallazgo poco frecuente, en ocasiones accidental, que se puede observar en cualquier nivel del tracto digestivo e inclusive fuera del mismo; es congénita y su patogenia está en discusión. Aunque su forma de presentación es lenta y progresiva y su comportamiento benigno y asintomático, en ocasiones puede originar manifestaciones obstructivas, hemorrágicas, inflamatorias y neoplásicas. En este último caso es imprescindible el diagnóstico histopatológico. Se presenta el caso de un paciente sintomático con heterotopia pancreática a nivel del píloro gástrico cuya localización es bastante infrecuente.
\end{abstract}

\section{Palabras clave \\ Páncreas heterotópico, pólipo gástrico.}

\begin{abstract}
Pancreatic heterotopia is rare and is sometimes found accidentally. It can occur anywhere in the digestive tract and even outside of it. Heterotopic pancreas is congenital, but its pathogenesis is under discussion. Although it develops slowly and progressively, its behavior is benign and asymptomatic. Nevertheless, it can manifest in obstructions, hemorrhaging, inflammation and neoplasia. In the latter case, histopathological diagnosis is essential. We present the case of a symptomatic patient with pancreatic heterotopia at the gastric pylorus, a very uncommon location.
\end{abstract}

Keywords

Heterotopic pancreas, gastric polyp.

\section{INTRODUCCIÓN}

El páncreas heterotópico o ectópico (PH) se caracteriza por la presencia de tejido pancreático normal en una estructura o sitio anatómico distinto sin continuidad vascular, neuronal ni anatómica con la glándula pancreática (1). Los autores consultados señalan a Shultz como responsable de la primera descripción del PH en 1727, también coinciden en que Klob es el primero en ofrecer la confirmación histopatológica en 1859; mientras que a Otschkin le adjudican en 1916 el primer informe de PH en la vesícula biliar (2). La frecuencia de este hallazgo se calcula entre el 0,11\%-13,7\%, es incidental en estudios de autopsia o durante procedimientos quirúrgicos y raramente de manera intencionada por la búsqueda de alguna enfermedad relacionada con la misma (3). Los sitios de localización más frecuentes son el duodeno (30,3\%), estómago $(26,5 \%)$, yeyuno (16,3\%), íleon ( $5,8 \%)$ y divertículo de Meckel (5,3\%). También se presenta en la vesícula biliar, vías biliares extrahepáticas, ampolla de Vater, mesenterio, vejiga, pulmón, esófago, tiroides, colón y bazo (3).

Von Heinrich (4) clasificó esta entidad por su aspecto morfológico en tres variedades: tipo I, cuando posee todos 
los elementos del páncreas normal; tipo II, en ausencia de islotes pancreáticos; y tipo III, solo se observan conductos rodeados de fascículos entrelazados de músculo liso. Este último tipo es conocido como adenomioma o hamartoma mucoepitelial (3). Se han señalado otras clasificaciones como la de Bromberg y colaboradores (5) en 2010, que ha tenido múltiples adeptos.

Aunque las características ecográficas endoscópicas típicas del páncreas ectópico incluyen ecogenicidad heterogénea, bordes indistintos y una ubicación dentro de 2 o más capas, también puede exhibir ecogenicidad homogénea hipoecoica y un borde distinto dentro de la cuarta capa ecográfica (muscular propia) similar a la ecografía endoscópica, característica de los tumores del estroma gastrointestinal (6).

A pesar del desarrollo de procedimientos diagnósticos modernos como el ultrasonido transendoscópico, la tomografía axial computarizada (TAC) y la gastroduodenoscopia, el diagnóstico sigue siendo difícil.

Los pacientes asintomáticos con diagnóstico positivo deben permanecer bajo supervisión médica y ser revisados periódicamente. Los pacientes sin complicaciones sintomáticos deben tener sus lesiones extirpadas, preferiblemente por resección local. En sitios accesibles para el fibroscopio, en manos experimentadas y en instituciones adecuadas, la extirpación endoscópica puede realizarse satisfactoriamente en pacientes seleccionados. El uso de la ecografía endoscópica en este tipo de intervención es imprescindible (7).

Las complicaciones descritas en $\mathrm{PH}$ son similares a aquellas que le ocurren al páncreas normal e incluyen pancreatitis aguda, cáncer pancreático, insulinomas, gastrinomas y degeneración quística $(2,3,8,9)$.

El objetivo de este trabajo es presentar el caso de un paciente que presentó manifestaciones digestivas altas refractarias al tratamiento convencional que motivó la realización de endoscopia digestiva en la que se encontró un engrosamiento polipoide gástrico, y por estudios histopatológicos se confirmó la presencia de una heterotopia pancreática, que es extremadamente infrecuente.

\section{CASO CLÍNICO}

Es un paciente de 66 años, masculino, jubilado, con antecedentes de artrosis degenerativa que se estaba tratando con antiinflamatorios no esteroideos (AINE) a dosis máxima. Acudió al hospital por dolor abdominal tipo ardor en el epigastrio de dos meses de evolución y se exacerbaba con la ingestión de alimentos, se acompañaba de ardor retroesternal y regurgitación gastroesofágica. Se valoró en conjunto con la especialidad de gastroenterología y se concluyó con el diagnóstico de gastropatía por los AINE, por lo que se comenzó el tratamiento con inhibidores de la bomba de protones (IBP) y agonistas dopaminérgicos. 20 días después acudió nuevamente por persistencia de la sintomatología, sin presentar mejoría alguna.

\section{Examen físico}

Obeso, piel y mucosas sin alteraciones, tejido celular subcutáneo (TCS) no infiltrado.

\section{Sistema cardiorrespiratorio}

Frecuencia respiratoria (FR): 19 respiraciones por minuto (rpm), murmullo vesicular normal, sin estertores, frecuencia cardíaca (FC) central: 96 latidos por minuto (lpm), tensión arterial (TA): 125/85 mm Hg.

\section{Sistema digestivo}

Abdomen globuloso, blando, depresible, doloroso a la palpación profunda en el epigastrio y mesogastrio; sin visceromegalia, sin masas abdominales y sin soplos abdominales.

El resto del examen físico no presentó alteraciones.

\section{Estudios analíticos}

Hemoglobina $(\mathrm{Hb}) 125 \mathrm{~g} / \mathrm{L}$, leucocitos $9 \times 10^{9} \mathrm{~g} / \mathrm{L}$ con fórmula diferencial normal. Velocidad de sedimentación globular $55 \mathrm{~mm} /$ primera hora. El colesterol, triglicéridos, glucemia, ácido úrico, urea, creatinina, proteínas totales y fraccionadas, y hepatograma resultaron normales. Por políticas del hospital se indicaron, además, se realizaron la prueba serológica para la sífilis (VDRL), que resultó no reactivo, y la del virus de la inmunodeficiencia humana (VIH; ELISA), que fue negativa.

La radiografía de tórax resultó normal y el ultrasonido abdominal con hígado de ecogenicidad difusa sin dilatación de las vías biliares. El páncreas no tenía alteraciones. Los riñones eran de tamaño normal, sin litiasis ni dilatación. La vejiga y próstata no tenían alteraciones. La radiografía de esófago, estómago y duodeno no fue concluyente para el diagnóstico.

\section{Esofagogastroduodenoscopia (panendoscopia)}

Estómago: en la región del antro se encontró un engrosamiento polipoide de $1,5 \mathrm{~cm}$ aproximadamente del mismo color que la mucosa circundante, exofítico sin presencia de erosiones con depresión central por la salida de conducto (Figura 1). Se procedió a tomar la biopsia de la lesión que informó la presencia de una heterotopia pancreática tipo I (Figuras 2 y 3). Esófago: primera y segunda porción del duodeno sin alteraciones.

Luego de realizado el diagnóstico de la enfermedad se modificaron las dosis de los medicamentos, se suspendieron los AINE y se fijó la fecha para el tratamiento quirúr- 
gico. Teniendo en cuenta el resultado histopatológico en esta asociación y la persistencia de los síntomas, se practicó la extirpación del área con el segmento de la pared gástrica en el que estaba implantado el tejido ectópico, resecando 3 $\mathrm{cm}$ de tejido sano a su alrededor.

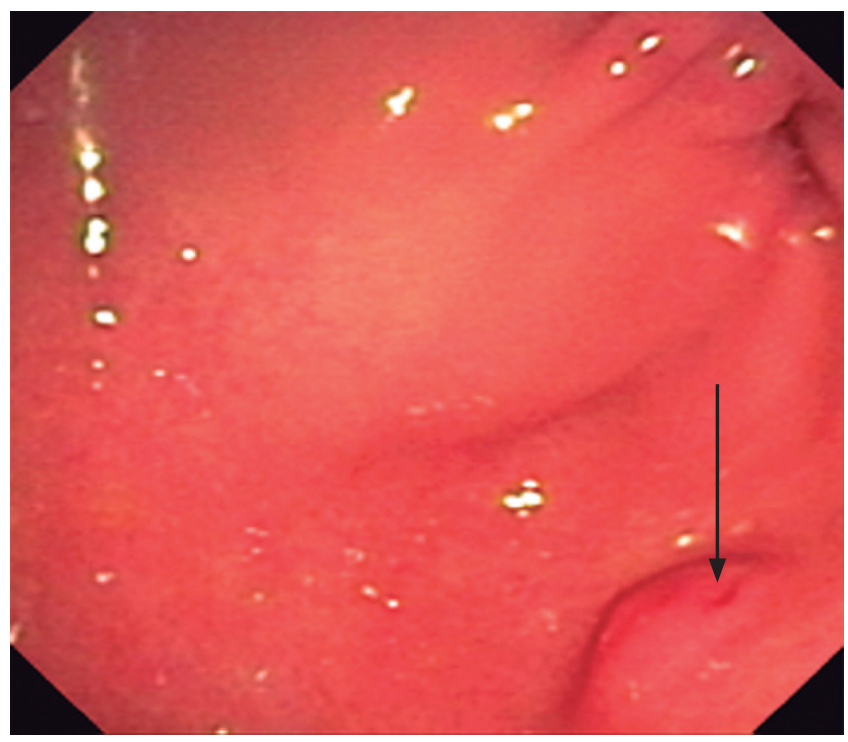

Figura 1. Imagen endoscópica del antro. Lesión submucosa que eleva la superficie simulando un tumor a nivel del antro. Obsérvese la pequeña depresión por la salida del conducto.

\section{DISCUSIÓN}

Se trata de una enfermedad ligada a la organogénesis que, aunque suele descubrirse en la edad adulta, se encuentra presente desde el nacimiento normalmente como una entidad benigna y asintomática. Predomina en varones, aunque es más común en el sexo femenino en edades pediátricas y en caso de $\mathrm{PH}$ de vesícula biliar $(3,5)$; este paciente era masculino y tenía 66 años.

En cuanto a la patogénesis, han surgido varias hipótesis para tratar de explicar este fenómeno, surgiendo interpretaciones que orientan hacia un origen hamartomatoso, tales como hiperplasia glandular focal, cambios reactivos en respuesta a un estímulo lesivo persistente, invaginación diverticular o involución fibroadenomatosa asociada con la vejez (10).

Las citoqueratinas detectadas por inmunohistoquímica en el adenomioma son similares a las que exhiben los sistemas ductal biliar y pancreático normal con positividad para citoqueratina 7 y negatividad para citoqueratina 20 (CK 7 +/CK 20-), por lo que al comparar este perfil de expresión con el que manifiesta el epitelio intestinal se observa un patrón invertido (CK 7 -/CK 20+), demostrado por Takahashi y Fukusato (8), con base en los trabajos previos

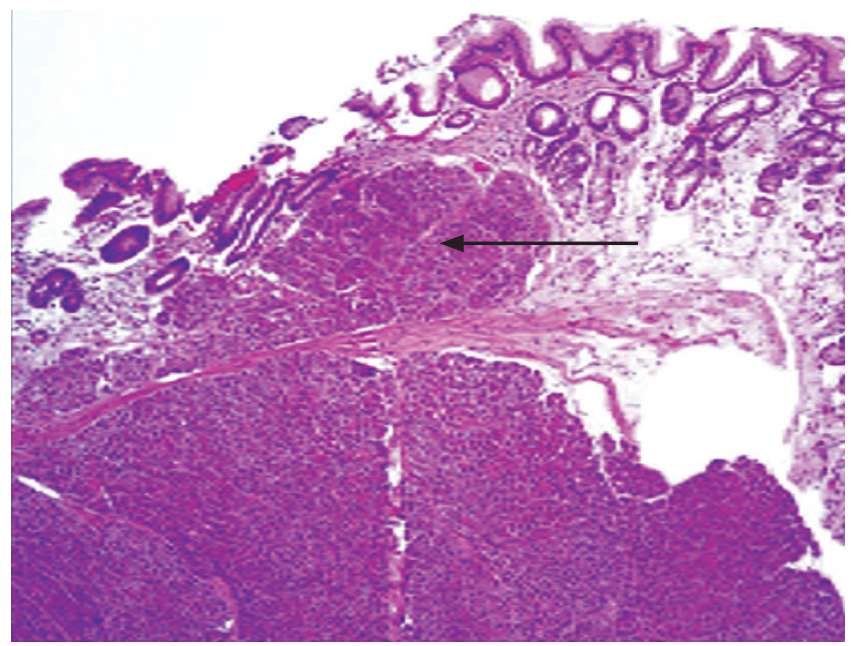

Figura 2. Imagen panorámica. Obsérvese la lesión bien delimitada a nivel de la submucosa y muscularis mucosae. H/E, $20 \mathrm{X}$.

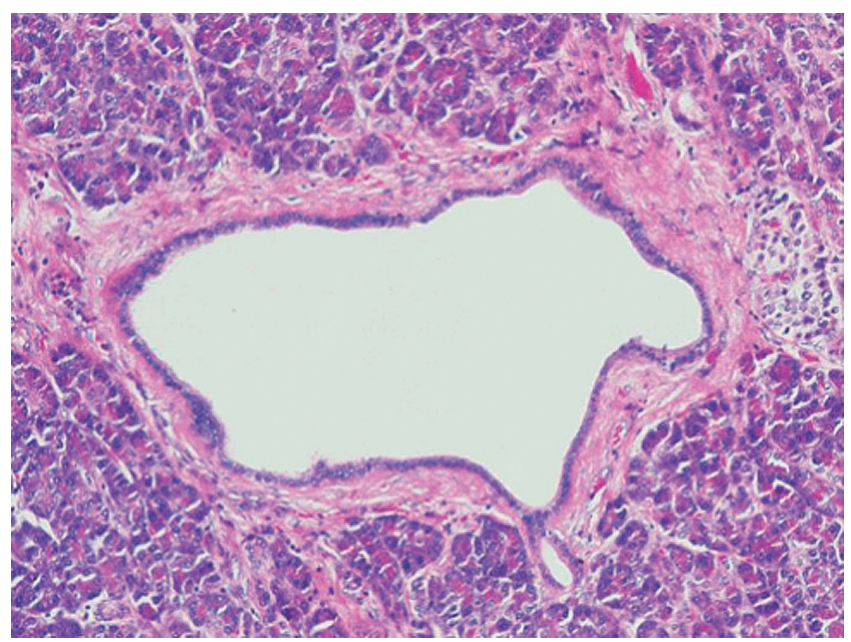

Figura 3. Imagen a mayor aumento típica: heterotopia tipo I. Obsérvense los conductos, ácinos y pequeños nidos de islotes hacia la derecha. H/E, $40 \mathrm{X}$.

de Babal, Handra-Luca y Yao (9-11). Macroscópicamente se le encuentra como un nódulo bien delimitado, de consistencia firme, con un tamaño que oscila de $1 \mathrm{~mm}$ a $5 \mathrm{~cm}$, único -en nuestro caso presentaba un tamaño de $1,5 \mathrm{~cm}$ - o múltiples, ubicado a cualquier nivel de la pared visceral, aunque su localización es submucosa, cuya superficie de corte es blanca-amarilla; sólida o quística, que desemboca hacia el lumen -como se observó en nuestro paciente-; y es mayor cuando se asocia con el divertículo de Meckel (11). Histológicamente, se distinguen los componentes del sistema ductal exocrino y endocrino en diferentes proporciones y combinaciones, como en nuestro paciente. 
La mayoría de los $\mathrm{PH}$ cursa asintomática, raramente se reconocen preoperatoriamente y se descubren durante la cirugía y el estudio histológico de la pieza anatómica, la exploración abdominal o durante la necropsia (12). En los casos de diagnóstico confirmado en pacientes asintomáticos, la recomendación varía de acuerdo con la localización y criterios de resecabilidad; teniendo siempre en cuenta su potencial de malignización (11). En ocasiones se indica una espera vigilada, pero si se tratara un hallazgo quirúrgico incidental, si es posible se aconseja realizar una resección completa transoperatoria, y de este modo se evitan complicaciones tardías y la necesidad de reoperación (12).

Alrededor del $30 \%-40 \%$ de los $\mathrm{PH}$ pueden dar síntomas gastrointestinales como en este caso, o producir complicaciones como pancreatitis aguda, cáncer pancreático, insulinoma, gastrinomas y degeneración quística (9). El diagnóstico diferencial debe realizarse con leiomiomas, linfomas, tumor neuroendocrino, tumor de estroma gastrointestinal y lesiones metastásicas $(2,3)$.

Los principales estudios diagnósticos incluyen la gastroduodenoscopia, la TAC y el ultrasonido endoscópico (este último no disponible para nosotros en el momento del estudio), que suele ser un recurso muy útil para detectar tumores pequeños menores de $2 \mathrm{~cm}$, pero no es específico: no puede excluir otras patologías tales como carcinoides, fibromas, granulomas eosinofílicos o leiomiomas. En los pacientes sintomáticos, habitualmente se requiere la exploración quirúrgica para un diagnóstico definitivo y para excluir otro tipo de lesiones, entre ellas las neoplásicas.

\section{CONCLUSIONES}

El PH se diagnostica con poca frecuencia. Este se descubre habitualmente en el duodeno, estómago, yeyuno, íleon y divertículo de Meckel; aunque puede tener otras localizaciones fuera del tubo digestivo. El diagnóstico definitivo es a menudo difícil y en este caso se confirmó por estudios histopatológicos.

\section{Conflictos de interés}

Los autores declaran no haber recibido ayuda económica o de cualquier tipo para la realización de este trabajo.

\section{REFERENCIAS}

1. Yuan Z, Chen J, Zheng Q, Huang XY, Yang Z, Tang J. Heterotopic pancreas in the gastrointestinal tract. World
J Gastroenterol. 2009;15(29):3701-3. doi: 10.3748/ wjg.15.3701.

2. Watanabe M, Shiozawa K, Kishimoto Y, Arai T, Nakano S, Kikuchi Y, et al. Heterotopic Pancreas of the Jejunum Incidentally Detected by Preoperative Abdominal CT: Report of Two Cases and Review of the Literature. Case Rep Gastroenterol. 2012;6(3):576-82. doi: 10.1159/000343093.

3. Karpińska MS, Nienartowicz M, MarkowskaWoyciechowska A, Budrewicz-Czapska K. Heterotopic pancreas in the stomach (type II according to Heinrich) - literature review and case report. Pol Przegl Chir. 2011;83(3):171-4. doi: 10.2478/v10035-011-0026-4.

4. Von Heinrich H. Beitrag Zur Histologie des Sogen: akzessorischen Pankreas. Virchows Arch A Pathol Anat Histhopathol. 1909;198:392-401. doi: 10.1007/ BF02085327.

5. Bromberg S, Neto C, Borges A. Heterotopia pancreática: análisis clínico patológico de 18 pacientes. Rev Col Bras Cir. 2010;37(6):413-9. doi: 10.1590/S010069912010000600007.

6. Chou JW, Cheng KS, Ting CF, Feng CL, Lin YT, Huang $\mathrm{WH}$. Endosonographic features of histologically proven gastric ectopic pancreas. Gastroenterol Res Pract. 2014;2014:160601. doi: 10.1155/2014/160601.

7. Bromberg SH, Camilo Neto C, Borges AF, Franco MI, França LC, Yamaguchi N. Pancreatic heterotopias: clinicopathological analysis of 18 patients. Rev Col Bras Cir. 2010;37(6):413-9.

8. Takahashi Y, Fukusato T. Adenomyoma of the small intestine. World J Gastrointest Pathophysiol. 2011;2(6):88-92. doi: 10.4291/wjgp.v2.i6.88.

9. Babál P, Zaviacic M, Danihel L. Evidence that adenomyoma of the duodenum is ectopic pancreas. Histopathology. 1998;33(5):487-8. doi: 10.1046/j.13652559.1998.0491d.x.

10. Handra-Luca A, Terris B, Couvelard A, Bonte H, Flejou JF. Adenomyoma and adenomyomatous hyperplasia of the Vaterian system: clinical, pathological, and new immunohistochemicalfeatures of 13 cases. ModPathol.2003;16(6):5306. doi: 10.1097/01.MP.0000073525.71096.8F.

11. Yao JL, Zhou H, Roche K, Bangaru BS, Ginsburg H, Greco MA. Adenomyoma arising in a meckel diverticulum: case report and review of the literature. Pediatr Dev Pathol. 2000;3(5):497-500. doi: 10.1007/s100240010097.

12. Lasky Davio M, Melgoza C, Benbassat M, Rescala E, Baquera J. Páncreas heterotópico de la vesícula biliar. AMCE. 2004,5(2):107-9. 